\title{
Entropy-Driven Chiral Order in a System of Achiral Bent Particles
}

\author{
Cristina Greco* and Alberta Ferrarini ${ }^{\dagger}$ \\ Dipartimento di Scienze Chimiche, Università di Padova, via Marzolo 1, 35131 Padova, Italy
}

(Received 2 June 2015; published 29 September 2015)

\begin{abstract}
Why should achiral particles organize into a helical structure? Here, using theory and molecular dynamics simulations we show that at high concentration crescent-shaped particles interacting through a purely repulsive potential form the twist-bend nematic phase, which features helical order of the twofold symmetry axes of particles, with doubly degenerate handedness. Spontaneous breaking of the chiral symmetry is driven by the entropic gain that derives from the decrease in excluded volume in the helical arrangement. Crucial to this purpose is the concave shape of particles. This study is based on a general formulation of the Onsager theory, which includes biaxiality and polarity of phase and particles, in addition to the space modulation of order. Molecular dynamics simulations corroborate the theoretical predictions and provide further insights into the structure of the helical phase.
\end{abstract}

DOI: 10.1103/PhysRevLett.115.147801

PACS numbers: 61.30.-v, 61.20.Ja, 61.30.St, 64.70.mf

The interactions and mechanism that control the propagation of chirality from the microscopic to the meso- and macroscopic level remain largely unexplained. Even more mysterious is the origin of chiral symmetry breaking in the absence of microscopic chirality. An intriguing case is that of bent-shaped molecules, which have been found to exhibit a variety of chiral liquid crystal phases, despite their intrinsic achirality [1-3]. The most recently discovered is the twist-bend nematic $\left(N_{\mathrm{TB}}\right)$ phase, where molecules organize into a helical superstructure, with pitch in the nanometer range [4-6] [see Fig. 1(b)]. This phase is nematic, in that the centers of mass of the molecules are randomly distributed, but features orientational order. The chiral symmetry of the phase is broken and the ground state has doubly degenerate handedness, with twin domains of opposite twist. The question then arises: Why do bent particles organize in a helical superstructure? If the reason is that at high density their packing has to optimally fill the space, it would seem more natural for them to arrange, for instance, into a structure like that sketched in Fig. 1(a). Another question is whether a bent shape of particles is sufficient for the spontaneous breaking of chiral symmetry. In the field of bent-shaped (thermotropic) liquid crystals a concept that has reached increasing consensus is that of "conformational deracemization" [7]: by virtue of their internal flexibility molecules, though being achiral, exhibit chiral conformations, and enantiomeric structures would spontaneously segregate into domains of opposite chirality.

To address this problem we have investigated a system of crescent-shaped particles, interacting via a purely repulsive potential. This minimalist choice allows us to explore what the basic requirements are for the onset of helical order and to get insights into the origin and structure of the $N_{\mathrm{TB}}$ phase. We have addressed this problem using an Onsagerlike theory [8], supplemented by molecular dynamics (MD) simulations, which confirm the theoretical results and give further structural insights into the helical organization of the system.

The seminal work of Onsager [8] demonstrated that at high concentration a system of hard spherocylinders, without any attractive interaction, forms a nematic phase and the driving force is the gain in translational entropy, related to the lower excluded volume in the ordered phase. Since then, there have been increasing theoretical, computational and experimental claims of entropy driven phase transitions (see Refs. $[9,10]$ for recent reviews). In general simple convex particles, like spherocylinders, were considered. Axial symmetry represents a considerable advantage for theory, because of the computational complexity introduced by additional degrees of freedom. To overcome this difficulty some tricks are generally used in the case of

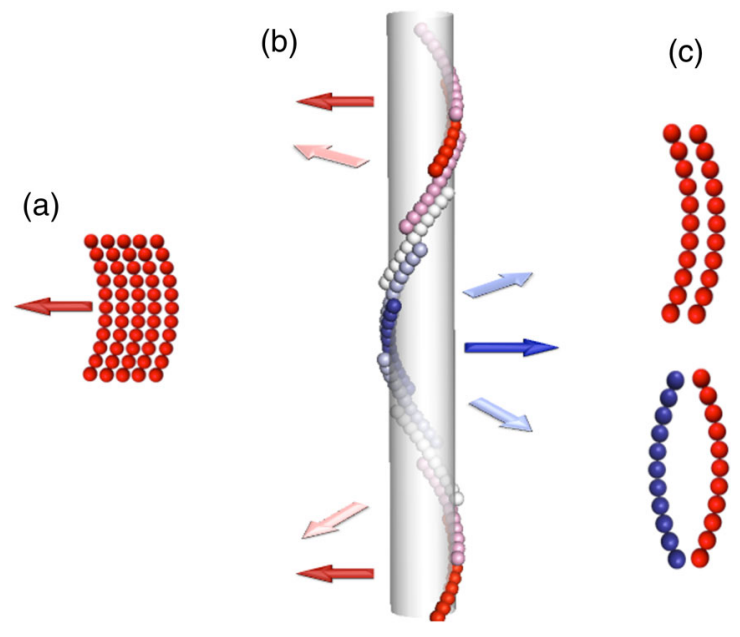

FIG. 1 (color online). Polar orthorombic (a) and helical (b) organization of bent particles. The arrows indicate the orientation of the twofold symmetry axes of particles. (c) Pair configurations where particles have parallel (top) and antiparallel (bottom) $C_{2}$ axes. 
biaxial particles; e.g., only a restricted set of orientations is sampled or some rotations are frozen. Here, we have developed a general formulation of the Onsager theory, which includes the biaxiality and polarity of the particles and phase, in addition to the spatial modulation of order.

In the framework of the Onsager theory the Helmholtz free energy of the system is expressed as a functional of the single particle density function and the second virial approximation is used for the excess contribution [8]. The $N_{\text {TB }}$ organization features helical periodicity, with pitch $p$, or wave vector $q=2 \pi / p$, with respect to an axis ( $Z$ axis of LAB frame, see Fig. 2). If we denote by $\Omega$ the Euler angles that define the particle orientation in the LAB frame, we can write $\rho(\Omega, Z)=\rho(Z) f(\Omega \mid Z)$, with $\rho(Z)$ the single particle positional density and $f(\Omega \mid Z)$ the single particle orientational distribution function at the $Z$ coordinate. The former is assumed to be uniform, $\rho(Z)=\bar{\rho}$, where $\bar{\rho}=1 / v=N / V$ is the average number density, with $N$ the number of particles and $V$ the volume of the sample. The orientational distribution function $f(\Omega \mid Z)$ is normalized as $\int d \Omega f(\Omega \mid Z)=1$, irrespective of $Z$. The Helmholtz free energy of the system can be expressed as the functional $A[\rho]$, with $A=A^{\text {id }}+A^{\text {or }}+A^{\text {ex }}$, where $A^{\text {id }}$ is the ideal gas free energy [11], $A^{\text {or }}$ accounts for the orientational entropy and $A^{\mathrm{ex}}$ is the excess term. The second contribution is given by

$$
\frac{A^{\text {or }}}{N k_{B} T}=\int d \Omega f(\Omega \mid Z) \ln \left[8 \pi^{2} f(\Omega \mid Z)\right]
$$

with $k_{B}$ the Boltzmann constant and $T$ the absolute temperature. The excess free energy is expressed in terms of the excluded volume between pairs of particles $(A, B)$ :
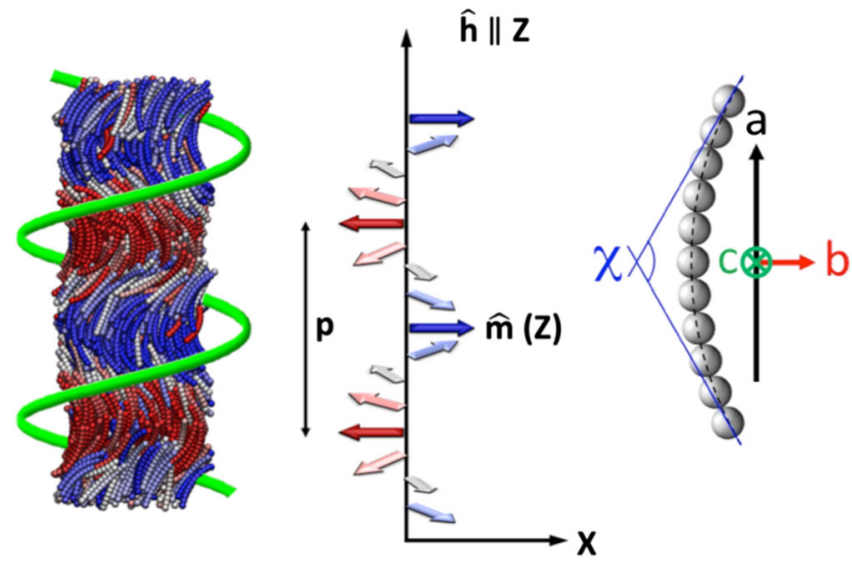

FIG. 2 (color online). Left: Helical structure of the $N_{\text {Тв }}$ phase. A snapshot from a MD trajectory is shown, with color codified according to the projection of the $C_{2}$ axes of particles on the $X$ axis of the LAB frame (blue-parallel, red-antiparallel). Middle: LAB frame $(X, Z)$. The unit vector $\hat{\mathbf{m}}$, parallel to the local twofold symmetry axis of the phase, performs a helical rotation with pitch $p$ around the unit vector $\hat{\mathbf{h}}$, parallel to the LAB $Z$ axis. Right: A crescent-shaped particle and the unit vectors $\hat{\mathbf{a}}$, $\hat{\mathbf{b}}, \hat{\mathbf{c}}$, attached to it.

$$
\begin{aligned}
\frac{A^{\mathrm{ex}}}{N k_{B} T}= & -\frac{G(\phi)}{2} \bar{\rho} \int d \Omega_{A} f\left(\Omega_{A} \mid Z_{A}=0\right) \\
& \times \int d \Omega_{B} \int d \mathbf{R}_{B} f\left(\Omega_{B} \mid Z_{B}\right) e_{A B}\left(\Omega_{A}, \Omega_{B}, \mathbf{R}_{B}\right),
\end{aligned}
$$

where $\mathbf{R}_{B}=\left(X_{B}, Y_{B}, Z_{B}\right)$ is the vector position of the $B$ particle in a frame with its origin at the position of the $A$ particle, and $e_{A B}$ is the Mayer function [11]. The function $G(\phi)=(1 / 4)(4-3 \phi) /(1-\phi)^{2}$, with the volume fraction $\phi=v_{0} / v$ where $v_{0}$ is the volume of a particle, is a correction accounting for the neglected higher order terms in the virial expansion $[12,13]$. The theoretical derivation is outlined in the Supplemental Material [14], and only the salient points are highlighted here. Given the thermodynamic conditions, the equilibrium orientational distribution function $f(\Omega \mid Z)$ is obtained by minimization of the free energy functional. Crucial to this purpose is the definition of a suitable functional form of $f(\Omega \mid Z)$. We have used its expansion on a basis of Wigner rotation matrices $\mathcal{D}_{M, N}^{J}(\Omega)[19,20]$ : $f(\Omega \mid Z)=\left(1 / 8 \pi^{2}\right) \sum(2 J+1)\left\langle\mathcal{D}_{M, N}^{J}(Z)\right\rangle_{\mathrm{LAB}} \mathcal{D}_{M, N}^{J}{ }^{*}(\Omega)$ [21], where the terms between angular brackets denote orientational averages and the subscript indicates that they are performed in the LAB frame. Taking advantage of the helical symmetry of the phase, we can write $f(\Omega \mid Z)=$ $\left(1 / 8 \pi^{2}\right) \sum(2 J+1)\left\langle\mathcal{D}_{M, N}^{J}\right\rangle e^{-i M q Z} \mathcal{D}_{M, N}^{J}{ }^{*}(\Omega)$, where $\left\langle\mathcal{D}_{M, N}^{J}\right\rangle$ are local order parameters. The $N_{\mathrm{TB}}$ phase has global $D_{\infty}$ symmetry, with the $C_{\infty}$ axis parallel to the helical axis ( $\hat{\mathbf{h}}$ in Fig. 2), and local $C_{2}$ symmetry, with the twofold axis ( $\hat{\mathbf{m}}$ in Fig. 2) perpendicular to $\hat{\mathbf{h}}$. Thus, for bent particles of $C_{2 v}$ symmetry, the nonvanishing order parameters are $\left\langle\mathcal{D}_{M, N}^{J}\right\rangle$ with either even $J, N$ or odd $J, N$ values and $M \neq 0$ in the latter case. The isotropic $(I)$ and the nematic $(N)$ phases, uniaxial and biaxial, are recovered as particular cases. In isotropic liquids all order parameters vanish, whereas in nematics the only nonvanishing order parameters are those having even $J, N$ values, with the additional restriction $M=0$ in the uniaxial case.

Using the expansion of $f(\Omega \mid Z)$, Eq. (1) becomes

$$
\begin{aligned}
& A^{\text {or }} / N k_{B} T=\left(1 / 8 \pi^{2}\right) \sum(2 J+1)\left\langle\mathcal{D}_{M, N}^{J}\right\rangle \\
& \quad \times \int d \Omega^{\prime} \mathcal{D}_{M, N}^{J}{ }^{*}\left(\Omega^{\prime}\right) \ln \left[\sum(2 L+1)\left\langle\mathcal{D}_{K, I}^{L}\right\rangle \mathcal{D}_{K, I}^{L}{ }^{*}\left(\Omega^{\prime}\right)\right],
\end{aligned}
$$

where $\Omega^{\prime}$ are the Euler angles describing the particle orientation in a local frame, with $Z^{\prime} \| \hat{\mathbf{h}}$ and $X^{\prime} \| \hat{\mathbf{m}}$ at the position of the center of mass of the particle. In Eq. (3) there is no explicit $Z$ dependence; the orientational entropy is the same as in a hypothetical nematic phase having order parameters equal to the local order parameters in the $N_{\mathrm{TB}}$ phase. Conversely, the $A^{\text {ex }}$ contribution is different in the two cases, and the decrease in the excluded volume deriving from the rototranslational coupling (the preferred 
orientation of a particle changes as is translates along $Z$ ) leads to the stabilization of the $N_{\text {Тв }}$ phase with respect to the uniform nematic phase. The two pair configurations in Fig. 1(c) help to illustrate this concept: the excluded volume is smaller when the two particles have parallel $C_{2}$ axes, compared to the case of antiparallel axes. This is a consequence of the nonconvex shape of particles. The helically modulated phase may also be favored over a layered structure, because it does not have the additional entropic cost deriving from the positional order [compare Figs. 1(a) and 1(b)]. Something analogous was recently demonstrated for the screwlike nematic phase of hard helices [22], which however was a simpler case, at least conceptually, because chirality was inscribed in the particle morphology.

These considerations have been confirmed by calculations for the model shown in Fig. 2. Each particle is made of 11 rigidly connected identical hard beads of diameter $\sigma$, arranged along an arc of a circle with contour length (center-to-center) equal to $10 \sigma$ and angle between end tangents $\chi=130^{\circ}$, a value deemed appropriate for the formation of the $N_{\mathrm{TB}}$ phase [23]. To determine the equilibrium state at a given density, the free energy $A$ is minimized with respect to the order parameters at different $p$ values. In the $N_{\text {TB }}$ phase $A(p)$ exhibits minima at finite $p$, which are very shallow close to the transition and become more pronounced on moving farther. The results are invariant with respect to a change of sign of $p$ (doubly degenerate handedness). The biaxial nematic phase never corresponds to the absolute free energy minimum. Figure 3 (bottom) displays the order parameters $\left\langle P_{2}\right\rangle_{\mathbf{a}}=\left\langle\mathcal{D}_{0,0}^{2}\right\rangle=$ $(3 / 2)\left\langle(\hat{\mathbf{a}} \cdot \hat{\mathbf{h}})^{2}\right\rangle-1 / 2$ and $\left\langle P_{1}\right\rangle_{\mathbf{b}}=\left\langle\mathcal{D}_{1,1}^{1}\right\rangle-\left\langle\mathcal{D}_{1,-1}^{1}\right\rangle=\langle\hat{\mathbf{b}} \cdot \hat{\mathbf{m}}\rangle$,

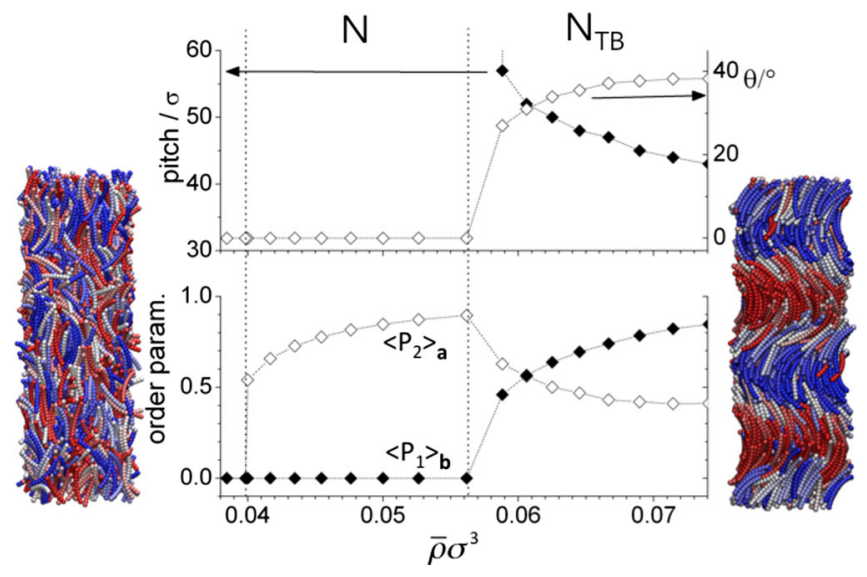

FIG. 3 (color online). Bottom: Order parameters $\left\langle P_{2}\right\rangle_{\mathbf{a}}$ (open) and $\left\langle P_{1}\right\rangle_{\mathbf{b}}$ (solid). Top: Helical pitch $p$ (solid) and conical angle $\theta$ (open), as a function of the number density $\bar{\rho}$, calculated for the particles in Fig. 2 using the Onsager-like theory. The images on the left and on the right are snapshots from MD simulations, in the $N$ and in the $N_{\text {TB }}$ phase, respectively; the color code is the same as in Fig. 2. as a function of the number density $\bar{\rho}$. The former is the nematic order parameter accounting for the (nonpolar) alignment of the particle $\mathbf{a}$ vectors to $\hat{\mathbf{h}}$, whereas the latter quantifies the polar order of $\hat{\mathbf{b}}$ vectors perpendicular to $\hat{\mathbf{h}}$. At low density both order parameters are equal to zero, the stable phase is isotropic. The jump of $\left\langle P_{2}\right\rangle_{\mathrm{a}}$ at $\bar{\rho}=0.04 / \sigma^{3}$ indicates the transition to the (uniaxial) $N$ phase. At higher density, the onset of helical organization is revealed by the appearance of transversal polar order and the simultaneous decrease of $\left\langle P_{2}\right\rangle_{\mathbf{a}}$. In usual descriptions of the $N_{\text {TB }}$ phase this decrease is ascribed to the tilt of the nematic director. Strictly speaking, in the $N_{\text {Тв }}$ phase there is only one local director defined by symmetry, which is $\hat{\mathbf{m}}$. The so-called "tilted director" [24], in a plane perpendicular to $\hat{\mathbf{m}}$, can be determined as the eigenvector corresponding to the major eigenvalue of the local $\mathbf{Q}$ tensor [25], quantifying the nematic ordering of the particle â vectors. Figure 3 (top) shows the tilt (or conical) angle $\theta$, between the tilted director and helical axis, together with the helical pitch $p$, as a function of the number density $\bar{\rho}$. On moving from the $N-N_{\mathrm{TB}}$ transition towards higher density, the pitch decreases until reaching values around $40 \sigma$, and simultaneously the tilt increases up to $\sim 38^{\circ}$.

To confirm the theoretical findings and get further insights into the helical organization we have carried out MD simulations. There were two previous claims of the $N_{\text {TB }}$ phase, both for thermotropic systems with attractive interparticle interactions. An early study, which can be taken as the first microscopic evidence of this phase, reported the appearance of the $N_{\mathrm{TB}}$ organization in a single trajectory of bent-shaped Gay-Berne particles [26]. In a more recent work this phase was detected in an all-atom simulation of a liquid crystal dimer, a relatively big molecule endowed with high flexibility [5]. There are no reports of an $N_{\mathrm{TB}}$ phase for bent particles with purely repulsive interactions. For rigid $V$-shaped particles nematic and smectic phases, even polar, were found [27,28], but chiral symmetry breaking was detected only in a crystal state [29]. We have carried out MD simulations for particles with the geometry shown in Fig. 2 (see the Supplemental Material [14] for details). Because of the need of a smooth, differentiable potential, the soft repulsive Weeks-ChandlerAndersen (WCA) form [30,31] has been assumed for the pair interaction between beads belonging to different particles. Simulations were run in the $(N, P, T)$ ensemble at $T=1.0 \epsilon / k_{B}$. At $\bar{\rho}=0.042 / \sigma^{3}$ the system exhibits a nematic phase, which beyond $\bar{\rho} \sim 0.050 / \sigma^{3}$ spontaneously organizes into a helical structure, whether right- or lefthanded. The simplicity of the model allows us to perform relatively long trajectories and to assess the stability of results: the helical phase organization is highly reproducible and robust with respect to the simulation conditions (system size and shape, starting conditions, etc.). The phase structure, easily recognizable by visual inspection (see snapshots in Fig. 3), was characterized through a suitable 


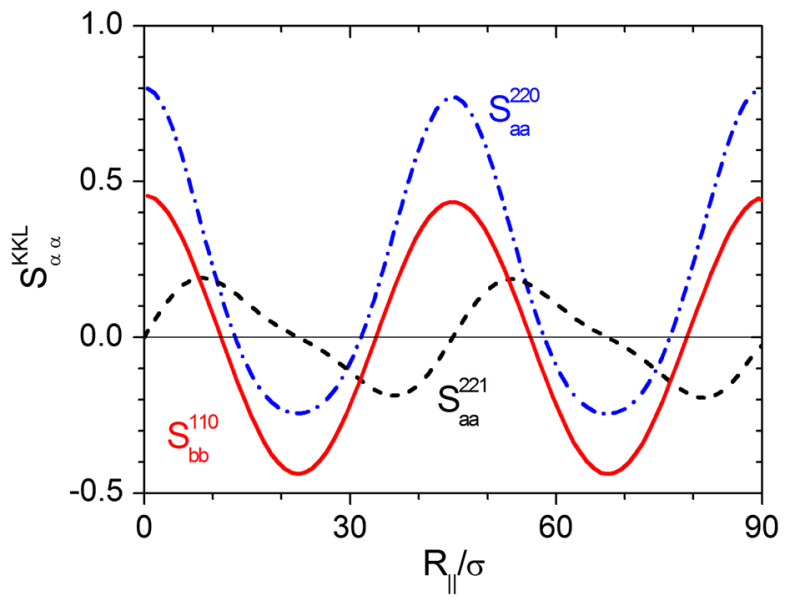

FIG. 4 (color online). Longitudinal orientational correlation functions from MD simulations at $\bar{\rho}=0.056 / \sigma^{3}$ (see text) as a function of $R_{\|}$, the interparticle distance along the helical axis: $S_{b b}^{110}$ (red solid line), $S_{a a}^{221}$ (black short-dashed line) and $S_{a a}^{220}$ (blue dash-dotted line).

set of orientational order parameters and pair correlation functions for the $\hat{\mathbf{a}}, \hat{\mathbf{b}}$ particle vectors (see Fig. 2). Figure 4 shows some of the correlation functions calculated for a system of $\mathcal{N}=3000$ particles, at $\bar{\rho}=0.056 / \sigma^{3}$ $\left(P=1.0 \epsilon / \sigma^{3}\right)$, in a box of height equal to $90 \sigma$, with periodic boundary conditions (PBCs). They are defined as [32]

$S_{b b}^{110}\left(R_{\|}\right)=\sum_{i=1}^{\mathcal{N}} \sum_{j=1, j \neq i}^{\mathcal{N}} \mathcal{C}_{i j}\left(\hat{\mathbf{b}}_{i} \cdot \hat{\mathbf{b}}_{j}\right)$

$S_{a a}^{221}\left(R_{\|}\right)=\sum_{i=1}^{\mathcal{N}} \sum_{j=1, j \neq i}^{\mathcal{N}} \mathcal{C}_{i j}\left[\left(\hat{\mathbf{a}}_{i} \times \hat{\mathbf{a}}_{j}\right) \cdot \frac{Z_{i j}}{\left|Z_{i j}\right|} \mathbf{Z}\right]\left(\hat{\mathbf{a}}_{i} \cdot \hat{\mathbf{a}}_{j}\right)$,

$S_{a a}^{220}\left(R_{\|}\right)=\sum_{i=1}^{\mathcal{N}} \sum_{j=1, j \neq i}^{\mathcal{N}} \mathcal{C}_{i j}\left[(3 / 2)\left(\hat{\mathbf{a}}_{i} \cdot \hat{\mathbf{a}}_{j}\right)^{2}-(1 / 2)\right]$,

where $\mathcal{C}_{i j}=\delta\left(\left|Z_{i j}\right|-R_{\|}\right) / \sum_{i^{\prime}=1}^{\mathcal{N}} \sum_{j^{\prime}=1, j^{\prime} \neq i^{\prime}}^{\mathcal{N}} \delta\left(\left|Z_{i^{\prime} j^{\prime}}\right|-R_{\|}\right)$ and $Z_{i j}$ is the distance between the $i$ and $j$ particle along the LAB $Z$ axis. $S_{b b}^{110}\left(R_{\|}\right)$accounts for polar orientational correlations between the $\hat{\mathbf{b}}$ vectors of particles having their center of mass on different planes perpendicular to $\hat{\mathbf{h}}$. This function vanishes in the $N$ phase and in a polar phase can take values between 1 (perfect parallel alignment) and -1 (perfect antiparallel alignment). The plot shows sinusoidal oscillations on moving along the $Z$ axis; this is a signature of the $N_{\mathrm{TB}}$ organization, where the particle $\hat{\mathbf{b}}$ axes spiral around $\hat{\mathbf{h}}$. The maxima (parallel correlations) and the minima (antiparallel correlations) are found for particles lying on planes at distances $n p$ and $(n+1 / 2) p$, respectively, with $n$ integer and $p=45 \sigma$. The helical organization and the pitch are confirmed by the behavior of $S_{a a}^{221}\left(R_{\|}\right)$, which is sensitive to the chirality of pair correlations between the â vectors of particles. The trend reported in Fig. 4 indicates a right-handed helix; the opposite sign is obtained for a left-handed helical arrangement. Finally, $S_{a a}^{220}\left(R_{\|}\right)$accounts for nematiclike orientational correlations between the â vectors. In the conventional $N$ phase this function takes a constant value, which reflects the degree of mutual alignment of the â vectors. In the $N_{\mathrm{TB}}$ phase the alignment direction rotates in a conical way on moving along $\hat{\mathbf{h}}$, which leads to oscillation of $S_{a a}^{220}$. The minima are located at $(n+1 / 2) p$ and their depth is related to the (tilt) angle between alignment direction and helical axis. Because of the PBCs, in simulations the helical pitch is constrained to be an integer fraction of the box dimension along the helix axis $L_{Z}$ [33]. By performing simulations in boxes with different values of $L_{Z}$, we could evaluate to what extent the helical pitch is influenced by the PBCs. A neat $N_{\text {TB }}$ structure, comprising $n$ pitches, formed only for $L_{Z}=n(45 \pm 5) \sigma$. This variability in the pitch is compatible with a weak $p$ dependence of the free energy in the neighborhood of its minima, predicted by the Onsager-like theory. Our MD results pose the question of why no $N_{\mathrm{TB}}$ phase was observed in simulations of repulsive $V$-shaped particles [27,28]. A possible reason could be that even relatively small changes in shape may influence the competition between the $N_{\mathrm{TB}}$ and other phases, in particular smectic ones. Further work will address this question.

In conclusion, using a generalized formulation of the Onsager theory and MD simulations we have demonstrated the spontaneous onset of helical ordering in a dense fluid of rigid crescent-shaped particles with purely repulsive mutual interactions. The system does not exhibit orthorombic order, although this is in principle possible, because of the entropic gain, related to the decrease of average excluded volume, in the helical organization. The general agreement between the results of theory and simulations suggests that the formation of the phase and its structural features are essentially determined by the size and shape of particles, with a special role played by their curvature and a minor influence of factors like the exact form of the interparticle potential or the inherent approximations of the theory. The development of an Onsager-like approach suitable for the $N_{\text {Тв }}$ phase requires the identification of the relevant order parameters and a theoretical framework accounting for them. In particular, recognition of the locally polar character of the helical order is crucial. We hope that our work will stimulate new theoretical and experimental investigation. Chiral symmetry breaking has been recently detected in 2D colloids [34] and its observation in 3D systems is challenging.

This work has been supported by MIUR (PRIN Contract 2010LKE4CC) and from Merck Chemicals Ltd (PhD scholarship to C. G.). We are grateful to Professor G. R. Luckhurst for stimulating discussions. 
*Present address: Max Planck Institute for Polymer Research, 55128 Mainz, Germany.

†alberta.ferrarini@unipd.it

[1] T. Niori, T. Sekine, J. Watanabe, T. Furukawa, and H. Takezoe, J. Mater. Chem. 6, 1231 (1996).

[2] D. Link, G. Natale, R. Shao, J. Maclennan, N. Clark, E. Körblova, and D. Walba, Science 278, 1924 (1997).

[3] H. Takezoe and Y. Takanishi, Jpn. J. Appl. Phys. 45, 597 (2006).

[4] M. Cestari, S. Diez-Berart, D. A. Dunmur, A. Ferrarini, M. R. de la Fuente, D. J. B. Jackson, D. O. Lopez, G. R. Luckhurst, M. A. Perez-Jubindo, R. M. Richardson, J. Salud, B. A. Timimi, and H. Zimmermann, Phys. Rev. E 84, 031704 (2011).

[5] D. Chen, J. H. Porada, J. B. Hooper, A. Klittnick, Y. Shen, M. R. Tuchband, E. Korblova, D. Bedrov, D. M. Walba, M. A. Glaser, J. E. Maclennan, and N. A. Clark, Proc. Natl. Acad. Sci. U.S.A. 110, 15931 (2013).

[6] V. Borshch, Y.-K. Kim, J. Xiang, M. Gao, A. Jákli, V. P. Panov, J. K. Vij, C. T. Imrie, M. G. Tamba, G. H. Mehl, and O. D. Lavrentovich, Nat. Commun. 4, 2635 (2013).

[7] D. J. Earl, M. A. Osipov, H. Takezoe, Y. Takanishi, and M. R. Wilson, Phys. Rev. E 71, 021706 (2005).

[8] L. Onsager, Ann. N.Y. Acad. Sci. 51, 627 (1949).

[9] L. Mederos, E. Velasco, and Y. Martínez-Ratón, J. Phys. Condens. Matter 26, 463101 (2014).

[10] M. Dijkstra, in Advances in Chemical Physics, edited by S. A. Rice and A. R. Dinner (Wiley, Hoboken, NJ, 2015), Vol. 156, pp. 35-71.

[11] D. A. McQuarrie, Statistical Mechanics (University Science Books, Sausalito, CA, 2000).

[12] J. D. Parsons, Phys. Rev. A 19, 1225 (1979).

[13] S. Lee, J. Chem. Phys. 87, 4972 (1987).

[14] See Supplemental Material http://link.aps.org/supplemental/ 10.1103/PhysRevLett.115.147801 for an outline of the derivation of the Onsager--like theory and for details on the molecular dynamics simulation protocol, which includes Refs. [15-18].

[15] S. Plimpton, J. Comp. Phys. 117, 1 (1995).
[16] H. Kamberaj, R. J. Low, and M. P. Neal, J. Chem. Phys. 122, 224114 (2005).

[17] G. J. Martyna, M. L. Klein, and M. Tuckerman, J. Chem. Phys. 97, 2635 (1992).

[18] G. J. Martyna, D. J. Tobias, and M. L. Klein, J. Chem. Phys. 101, 4177 (1994).

[19] C. Zannoni, in The Molecular Physics of Liquid Crystals, edited by G. R. Luckhurst and G. W. Gray (Academic Press, New York, 1979).

[20] R. N. Zare, Angular Momentum (Wiley, New York, 1988).

[21] To simplify the notation, here and henceforth it is implicitly assumed that summations run over all the indexes of Wigner rotation matrices as follows: $\sum \mathcal{D}_{M, N}^{J} \ldots \equiv$ $\sum_{J=0}^{\infty} \sum_{M=-J}^{J} \sum_{N=-J}^{J} \mathcal{D}_{M, N}^{J} \cdots$

[22] H. B. Kolli, E. Frezza, G. Cinacchi, A. Ferrarini, A. Giacometti, and T. S. Hudson, J. Chem. Phys. 140, 081101 (2014).

[23] C. Greco, G. R. Luckhurst, and A. Ferrarini, Soft Matter 10, 9318 (2014).

[24] I. Dozov, Europhys. Lett. 56, 247 (2001).

[25] P. G. de Gennes and J. Prost, The Physics of Liquid Crystals (Clarendon Press, Oxford, 1993).

[26] R. Memmer, Liq. Cryst. 29, 483 (2002).

[27] Y. Lansac, P. K. Maiti, N. A. Clark, and M. A. Glaser, Phys. Rev. E 67, 011703 (2003).

[28] A. Dewar and P. J. Camp, J. Chem. Phys. 123, 174907 (2005).

[29] J. Xu, R. L. B. Selinger, J. V. Selinger, and R. Shashidhar, J. Chem. Phys. 115, 4333 (2001).

[30] J. D. Weeks, D. Chandler, and H. C. Andersen, J. Chem. Phys. 54, 5237 (1971).

[31] J. D. Weeks, D. Chandler, and H. C. Andersen, Science 220, 787 (1983).

[32] These functions coincide, apart from a constant factor, with orientationally averaged Stone invariants, see A. J. Stone, Mol. Phys. 36, 241 (1978).

[33] D. Frenkel, Eur. Phys. J. Plus 128, 10 (2013).

[34] K. Zhao, R. Bruinsma, and T. G. Mason, Nat. Commun. 3, 801 (2012). 